FACTA UNIVERSITATIS (NIŠ)

Ser. Math. Inform. Vol. 34, No 4 (2019), 781-788

https://doi.org/10.22190/FUMI1904781S

\title{
FAULT-TOLERANT METRIC DIMENSION OF CIRCULANT GRAPHS
}

\author{
Narjes Seyedi and Hamid Reza Maimani*
}

(C) 2019 by University of Niš, Serbia | Creative Commons Licence: CC BY-NC-ND

\begin{abstract}
A set $W$ of vertices in a graph $G$ is called a resolving set for $G$ if for every pair of distinct vertices $u$ and $v$ of $G$, there exists a vertex $w \in W$ such that the distance between $u$ and $w$ is different from the distance between $v$ and $w$. The cardinality of a minimum resolving set is called the metric dimension of $G$, denoted by $\beta(G)$. A resolving set $W$ for $G$ is fault-tolerant if $W \backslash\{w\}$ is also a resolving set, for each $w$ in $W$. The fault-tolerant metric dimension of $G$ is the size of a smallest fault-tolerant resolving set for $G$, denoted by $\beta^{\prime}(G)$. In this paper, we study the fault-tolerant metric dimension of a family of circulant graphs $X_{n, 3}$ with connection set $C=\left\{1, \frac{n}{2}, n-1\right\}$, when $n$ is even and circulant graphs $X_{n, 4}$ with connection set $C=\{ \pm 1, \pm 2\}$.
\end{abstract}

Keywords. Circulant graphs; resolving set; fault-tolerant metric dimension.

\section{Introduction}

The metric dimension problem was introduced independently by Slater [15] and Harary and Melter [8]. The metric dimension arises in many diverse areas, including telecommunications [3], connected joints in graphs and chemistry [4], the robot navigation [12] and geographical routing protocols [13], etc.

For a connected graph $G$ with vertex set $V(G)$ and edge set $E(G)$, the distance between two vertices $u$ and $v$ in $V(G)$ is the number of edges in a shortest path connecting them, and is denoted by $d(u, v)$. Consider an ordered set $W=\left\{w_{1}, w_{2}, \cdots, w_{k}\right\} \subseteq V(G)$. For each $v \in V(G)$ the code of $v$ with respect to $W$ is $\left(d\left(v, w_{1}\right), d\left(v, w_{2}\right), \cdots, d\left(v, w_{k}\right)\right)$, denoted by $c_{W}(v)$. The set $W$ is called a resolving set for $G$, if all vertices of $G$ have distinct codes. The minimum cardinality of a resolving set of $G$ is called the metric dimension of $G$ and is denoted by $\beta(G)$. A resolving set of order $\beta(G)$ is called a metric basis of $G$ [2].

Elements of bases were referred to as sensors in an application given in [5]. If one

Received June 14, 2019; accepted August 16, 2019

2010 Mathematics Subject Classification. Primary 05C12

* Corresponding Author 
of the sensors does not work properly, we will not have enough information to deal with the intruder (fire, thief etc). In order to overcome this kind of problems, concept of fault-tolerant metric dimension was presented in [9]. Fault-tolerant resolving sets provide correct information even when one of the sensors is not working. A resolving set $W$ of a graph $\mathrm{G}$ is fault-tolerant if $W \backslash\{w\}$ is also a resolving set, for each $\mathrm{w}$ in $W$. The fault-tolerant metric dimension of $G$ is the minimum cardinality of a fault-tolerant resolving set, denoted by $\beta^{\prime}(G)$. A fault-tolerant resolving set of order $\beta^{\prime}(G)$ is called a fault-tolerant metric basis.

The circulant graph is a graph with vertex set $\mathbb{Z}_{n}$, an additive group of integers modulo $n$, and two vertices labeled $i$ and $j$ are adjacent if and only if $i-j(\bmod n) \in C$, where $C \subset \mathbb{Z}_{n}$, which is called connection set, has the property that $C=-C$ and $0 \notin C$. The circulant graph is denoted by $X_{n, \Delta}$ where $\Delta=|C|$.

Two graphs $G_{1}=\left(V_{1}, E_{1}\right)$ and $G_{2}=\left(V_{2}, E_{2}\right)$ are said to be isomorphic if there is a bijective mapping $f$ from $V_{1}$ to $V_{2}$ such that $u v \in E_{1}$ if and only if $f(u) f(v) \in E_{2}$. An automorphism of a graph is an isomorphism from the graph to itself. The set of all automorphisms of a graph, G, forms a group, denoted by $\operatorname{Aut}(G)$. It is well known that, if $G$ is a circulant graph, then $\mathbb{Z}_{n}$ is a subgroup of $A u t(G)$ [7].

In this paper, we consider a family of circulant graphs $X_{n, 3}$ with connection set $C=\left\{1, \frac{n}{2}, n-1\right\}$, when $n$ is even and prove that the fault-tolerant metric dimension of this family of graphs is independent of choice of $\mathrm{n}$ by showing that $\beta^{\prime}\left(X_{n, 3}\right)=4$, for all $n \geq 4$ and $n \equiv 0(\bmod 4)$, in Theorem 2.2 , and $\beta^{\prime}\left(X_{n, 3}\right) \leq 6$, for all $n \geq 10$ and $n \equiv 2(\bmod 4)$, in Theorem 2.4. We also consider a family of circulant graphs $X_{n, 4}$ with connection set $C=\{ \pm 1, \pm 2\}$ and prove that the fault-tolerant metric dimension of this family of graphs is independent of choice of $\mathrm{n}$ by showing that $\beta^{\prime}\left(X_{n, 4}\right)=4$, for all $n \geq 10$ and $n \equiv 2(\bmod 4)$, in Theorem 3.1 .

\section{Fault-Tolerant Metric Dimension Of Circulant Graphs $X_{n, 3}$}

Salman et al. [14] characterized the metric dimension for family of circulant graphs $X_{n, 3}$ with connection set $C=\left\{1, \frac{n}{2}, n-1\right\}$ for even $n$. Now we obtain the faulttolerant metric dimension of this family of graphs.

Theorem 2.1. [14, Theorem 2.2] Let $n$ be an integer and $n \equiv 0(\bmod 4)$. If $k=\frac{n}{4}$, then for any $1 \leq i \leq k$ the set $W=\left\{v_{i}, v_{i+1}, v_{i+2 k}\right\}$ is a resolving set and hence $\beta\left(X_{n, 3}\right)=3$.

The following lemma, gave a new family of resolving set of $X_{n, 3}$ of size 3 , where $n \equiv 0(\bmod 4)$.

Lemma 2.1. Let $n$ be an integer, $n \equiv 0(\bmod 4)$ and $k=\frac{n}{4}$. Then the set $W=$ $\left\{v_{i}, v_{i+1}, v_{i+2 k+1}\right\}$ is a resolving set of $X_{n, 3}$, for any $1 \leq i \leq k$.

Proof. Let $W=\left\{v_{i}, v_{i+1}, v_{i+2 k+1}\right\}$ for fixed i; $0 \leq i \leq k$ where $k=\frac{n}{4}$. We compute the codes of all $v \in V\left(X_{n, 3}\right) \backslash W$. We have

$$
c_{W}\left(v_{i+k}\right)=(k, k-1, k), c_{W}\left(v_{i+k+1}\right)=(k, k, k), c_{W}\left(v_{i+3 k}\right)=(k, k, k-1),
$$




$$
c_{W}\left(v_{i+3 k+1}\right)=(k-1, k, k), c_{W}\left(v_{i+2 k}\right)=(1,2,1) .
$$

The codes of other vertices are listed in Table 1. By a simple computing these codes are distinct and hence $W$ is a resolving set of $X_{n, 3}$.

Table 1

\begin{tabular}{|c|c|c|c|}
\hline Shortest paths between & $v_{i}$ & $v_{i+1}$ & $v_{i+2 k+1}$ \\
\hline$v_{i+j+1}(1 \leq j \leq k-2)$ & $j+1$ & $j$ & $j+1$ \\
\hline$v_{i+k+j}(2 \leq j \leq k-1)$ & $k-j+1$ & $k-j+2$ & $k-j+1$ \\
\hline$v_{i+2 k+j}(2 \leq j \leq k-1)$ & $j$ & $j-1$ & $j-1$ \\
\hline$v_{i+3 k+j}(2 \leq j \leq k-1)$ & $k-j$ & $k-j+1$ & $k-j+2$ \\
\hline
\end{tabular}

Theorem 2.2. For all $n \geq 4$ and $n \equiv 0(\bmod 4), \beta^{\prime}\left(X_{n, 3}\right)=4$.

Proof. From the definition of fault-tolerant metric dimension it can be seen that $\beta^{\prime}(G) \geq \beta(G)+1[11]$. This implies that $\beta^{\prime}\left(X_{n, 3}\right) \geq 4$ since $\beta\left(X_{n, 3}\right)=3$ by Theorem 2.1 .

Now for the lower bound, Let $W^{\prime}=\left\{v_{i}, v_{i+1}, v_{i+2 k}, v_{i+2 k+1}\right\}$ for fixed i; $0 \leq i \leq$ $k$ where $k=\frac{n}{4}$. We will show that for each $x \in W^{\prime}$, the set $W^{\prime} \backslash\{x\}$ is a resolving set for $X_{n, 3}$. At first note that $\mathbb{Z}_{n}$ is subgroup of $\operatorname{Aut}\left(X_{n, 3}\right)$ and if $f=\left(v_{o}, v_{1}, \cdots, v_{n-1}\right)$ is a cycle of order $n$, then $\mathbb{Z}_{n} \cong<f>$. In addition $f^{j}\left(v_{i}\right)=v_{i+j}$. Now we consider the following cases:

Case 1. Suppose that $x=v_{i}$. We have

$$
f^{j}\left(\left\{v_{i+1}, v_{i+2 k}, v_{i+2 k+1}\right\}\right)=\left\{v_{j+i+1}, v_{j+i+2 k}, v_{j+i+2 k+1}\right\}
$$

. If $j=2 k$, then

$$
f^{j}\left(\left\{v_{i+1}, v_{i+2 k}, v_{i+2 k+1}\right\}\right)=\left\{v_{i+2 k+1}, v_{i}, v_{i+1}\right\},
$$

and

$$
f^{j}\left(\left\{v_{i+2 k+1}, v_{i}, v_{i+1}\right\}\right)=\left\{v_{i+1}, v_{i+2 k}, v_{i+2 k+1}\right\} .
$$

By Lemma 2.1, $\left\{v_{i}, v_{i+1}, v_{i+2 k+1}\right\}$ is a resolving set for $X_{n, 3}$ and since automorphisms of graphs preserves the properties of the graph, we conclude that $W^{\prime} \backslash\{x\}=$ $\left\{v_{i+1}, v_{i+2 k}, v_{i+2 k+1}\right\}$ is a resolving set for $X_{n, 3}$.

Case 2. Let $x=v_{i+1}$. We have

$$
f^{j}\left(\left\{v_{i}, v_{i+1}, v_{i+2 k}\right\}\right)=\left\{v_{j+i}, v_{j+i+1}, v_{j+i+2 k}\right\} .
$$

If $j=2 k$, then

$$
f^{j}\left(\left\{v_{i}, v_{i+1}, v_{i+2 k}\right\}\right)=\left\{v_{i+2 k}, v_{i+2 k+1}, v_{i}\right\}
$$


. Hence By the same argument of Case 1, the set $W^{\prime} \backslash\{x\}=\left\{v_{i+2 k}, v_{i+2 k+1}, v_{i}\right\}$ is a resolving set for $X_{n, 3}$.

Case 3. If $x=v_{i+2 k}$, then according to the Lemma 2.1, $W^{\prime} \backslash\{x\}$ is a resolving set for $X_{n, 3}$.

Case 4. If $x=v_{i+2 k+1}$, then according to the Theorem 2.1, $W^{\prime} \backslash\{x\}$ is a resolving set for $X_{n, 3}$.

Therefore, $W^{\prime}$ is the fault-tolerant resolving set for this family of graphs. Thus $\beta^{\prime}\left(X_{n, 3}\right) \leq 4$, for all $n \geq 10$ and $n \equiv 2(\bmod 4)$. This completes the proof.

Now we study the fault-tolerant metric dimension of $X_{n, 3}$ in the case $n \equiv 2(\bmod 4)$.

Theorem 2.3. [14, Theorem 2.5] Let $n \geq 6$ be an integer and $n \equiv 2(\bmod 4)$. If $k=\frac{n-2}{4}$, then $W=\left\{v_{i}, v_{i+1}, v_{i+2 k}, v_{i+2 k+1}\right\}$ is a resolving set for $X_{n, 3}$ for any $1 \leq i \leq k$. In addition $\beta\left(X_{n, 3}\right)=4$.

In the following lemma we gave some resolving sets of size 3 for $X_{n, 3}$.

Lemma 2.2. Let $n \geq 10$ be an integer and $n \equiv 2(\bmod 4)$. For $k=\frac{n-2}{4}$ and any $1 \leq i \leq k$ the following sets are resolving sets of size 4 of $X_{n, 3}$,

i) $W_{1}=\left\{v_{i}, v_{i+1}, v_{i+2 k+1}, v_{i+2 k+2}\right\}$;

ii) $W_{2}=\left\{v_{i+1}, v_{i+2 k}, v_{i+2 k+2}, v_{i+4 k+1}\right\}$;

iii) $W_{3}=\left\{v_{i}, v_{i+2 k+1}, v_{i+2 k+2}, v_{i+4 k+1}\right\}$.

Proof. Suppose that $k=\frac{n-2}{4}$ and $W=\left\{v_{i}, v_{i+1}, v_{i+2 k+1}, v_{i+2 k+2}\right\}$ where $0 \leq i \leq k$ . We compute $c_{W_{1}}(v)$ for $v \in V\left(X_{n, 3}\right) \backslash W_{1}$. We have

$$
\begin{gathered}
c_{W_{1}}\left(v_{i+k}\right)=(k, k-1, k+1, k), c_{W_{1}}\left(v_{i+k+1}\right)=(k+1, k, k, k+1), \\
c_{W_{1}}\left(v_{i+2 k}\right)=(2,3,1,2), c_{W_{1}}\left(v_{i+3 k+2}\right)=(k, k+1, k+1, k) .
\end{gathered}
$$

The codes of other vertices respect to $W_{1}$, are shown in Table 2 . It is not difficult to see that all codes are distinct and hence $W_{1}$ is a resolving set of $X_{n, 3}$.

Table 2

\begin{tabular}{|c|c|c|c|c|}
\hline Shortest paths between & $v_{i}$ & $v_{i+1}$ & $v_{i+2 k+1}$ & $v_{i+2 k+2}$ \\
\hline$v_{i+j+1}(1 \leq j \leq k-2)$ & $j+1$ & $j$ & $j+2$ & $j+1$ \\
\hline$v_{i+k+j}(2 \leq j \leq k-1$ & $k-j+2$ & $k-j+3$ & $k-j+1$ & $k-j+2$ \\
\hline$v_{i+2 k+j+1}(2 \leq j \leq k)$ & $j+1$ & $j$ & $j$ & $j-1$ \\
\hline$v_{i+3 k+j+1}(2 \leq j \leq k)$ & $k-j+1$ & $k-j+2$ & $k-j+2$ & $k-j+3$ \\
\hline
\end{tabular}

For $W_{2}$ we have,

$$
c_{W_{2}}\left(v_{i+k}\right)=(k-1, k, k, k+1), c_{W_{2}}\left(v_{i+k+1}\right)=(k, k-1, k+1, k),
$$


Table 3

\begin{tabular}{|c|c|c|c|c|}
\hline Shortest paths between & $v_{i+1}$ & $v_{i+2 k}$ & $v_{i+2 k+2}$ & $v_{i+4 k+1}$ \\
\hline$v_{i+j+1}(1 \leq j \leq k-2)$ & $j$ & $j+3$ & $j+1$ & $j+2$ \\
\hline$v_{i+k+j}(2 \leq j \leq k-1$ & $k-j+3$ & $k-j$ & $k-j+2$ & $k-j+1$ \\
\hline$v_{i+2 k+j+1}(2 \leq j \leq k)$ & $j$ & $j+1$ & $j-1$ & $j$ \\
\hline$v_{i+3 k+j+1}(2 \leq j \leq k-1)$ & $k-j+2$ & $k-j+1$ & $k-j+3$ & $k-j$ \\
\hline
\end{tabular}

$$
c_{W_{2}}\left(v_{i+2 k}\right)=(2,1,1,2), c_{W_{2}}\left(v_{i+3 k+2}\right)=(k+1, k, k, k-1),
$$

and the codes of other vertices are listed Table 3. These codes are distinct and we conclude that $W_{2}$ is a resolving set for $X_{n, 3}$.

Similarly for $W_{3}$, we have

$$
\begin{gathered}
c_{W_{3}}\left(v_{1}\right)=(1,2,1,2), c_{W_{3}}\left(v_{i+k}\right)=(k, k+1, k, k+1), c_{W_{3}}\left(v_{i+k+1}\right)=(k+1, k, k+1, k), \\
c_{W_{3}}\left(v_{i+2 k}\right)=(2,1,2,1), c_{W_{3}}\left(v_{i+3 k+2}\right)=(k, k+1, k, k-1),
\end{gathered}
$$

and for other vertices, the codes are listed in Table 4 . By these codes, we conclude that $W_{3}$ is a resolving set for $X_{n, 3}$.

Table 4

\begin{tabular}{|c|c|c|c|c|}
\hline Shortest paths between & $v_{i}$ & $v_{i+2 k+1}$ & $v_{i+2 k+2}$ & $v_{i+4 k+1}$ \\
\hline$v_{i+j+1}(1 \leq j \leq k-2)$ & $j+1$ & $j+2$ & $j+1$ & $j+2$ \\
\hline$v_{i+k+j}(2 \leq j \leq k-1)$ & $k-j+2$ & $k-j+1$ & $k-j+2$ & $k-j+1$ \\
\hline$v_{i+2 k+j+1}(2 \leq j \leq k)$ & $j+1$ & $j$ & $j-1$ & $j$ \\
\hline$v_{i+3 k+j+1}(2 \leq j \leq k-1)$ & $k-j+1$ & $k-j+2$ & $k-j+3$ & $k-j$ \\
\hline
\end{tabular}

Theorem 2.4. For all $n \geq 6$ and $n \equiv 2(\bmod 4), \beta^{\prime}\left(X_{n, 3}\right) \leq 6$.

Proof. For $n=6, X_{6,3} \simeq K_{3,3}$. This implies that $\beta^{\prime}\left(X_{6,3}\right)=6$ since $\beta^{\prime}\left(K_{m, n}\right)=$ $m+n[6$, Proposition 1].

Now suppose that $n \geq 10$. Let $k=\frac{n-2}{4}$. For a fixed $i$, where $0 \leq i \leq k$, consider the set

$$
W=\left\{v_{i}, v_{i+1}, v_{i+2 k}, v_{i+2 k+1}, v_{i+2 k+2}, v_{i+4 k+1}\right\} .
$$

Since $W$ contains the set $W_{1}$ of Theorem $2.3(i)$, so $W$ is a resolving set for $X_{n, 3}$. Now we will show that for each $x \in W^{\prime}$, the set $W^{\prime} \backslash\{x\}$ is a resolving set for $X_{n, 3}$. We have the following cases:

Case 1. If $x \in\left\{v_{i}, v_{i+2 k+1}\right\}$, then $W \backslash\{x\}$ contains a set $W_{2}$ listed in Lemma 2.2 (ii). Thus $W \backslash\{x\}$ is a resolving set for $X_{n, 3}$.

Case 2. If $x=v_{i+1}$, then $W \backslash\{x\}$ contains a set $W_{3}$ listed in Lemma 2.2 (iii). So $W \backslash\{x\}$ is a resolving set for $X_{n, 3}$. 
Case 3. If $x=v_{i+2 k}$, then $W \backslash\{x\}$ contains a set $W$ listed in Lemma $2.2(i)$. Hence $W \backslash\{x\}$ is a resolving set for $X_{n, 3}$.

Case 4. If $x \in\left\{v_{i+2 k+2}, v_{i+4 k+1}\right\}$, then $W \backslash\{x\}$ contains a set $W^{\prime}$ listed in Theorem 2.3. Hence $W \backslash\{x\}$ is a resolving set for $X_{n, 3}$.

Therefore, $W$ is the fault-tolerant resolving set for this family of graphs. Thus $\beta^{\prime}\left(X_{n, 3}\right) \leq 6$, for all $n \geq 10$ and $n \equiv 2(\bmod 4)$.

\section{Fault-Tolerant Metric Dimension Of Circulant Graphs $X_{n, 4}$}

In this section consider $X_{n, 4}$ with connection set $C=\{ \pm 1, \pm 2\}$. In [1], Borchert and Gosselin showed that $\operatorname{dim}\left(X_{n, 4}\right)=4$ if $n=1(\bmod 4)$ and $\operatorname{dim}\left(X_{n, 4}\right)=3$ otherwise. Now we study the fault-tolerant metric dimension of this family of graphs in the case $n \equiv 2(\bmod 4)$.

In the following lemma we obtain some resolving sets for $X_{n, 4}$.

Lemma 3.1. Let $n \geq 10$ and $n \equiv 2(\bmod 4)$. For $k=\frac{n-2}{4}$ and any $1 \leq i \leq k$, the following sets are resolving sets for $X_{n, 4}$,

i) $W_{1}=\left\{v_{i}, v_{i+1}, v_{i+2}\right\}$;

ii) $W_{2}=\left\{v_{i}, v_{i+1}, v_{i+3}\right\}$;

iii) $W_{3}=\left\{v_{i}, v_{i+2}, v_{i+3}\right\}$.

Proof. The set $W_{1}$ is a resolving set by [10, Theorem 5]. For the parts (ii) and (iii), we prove that the sets $W_{2}$ and $W_{3}$ are resolving sets of $X_{n, 4}$ for $i=0$. The remaining cases, obtained by this fact that $\mathbb{Z}_{n}$ is a subgraph of $\operatorname{Aut}\left(X_{n, 4}\right)$. By a simple computing we can obtain the codes of vertices respect to $W_{2}$ and $W_{3}$. These codes listed in Table 5 and Table 6 . Clearly these codes are distinct and hence the sets $W_{2}$ and $W_{3}$ are resolving sets.

Table 5

\begin{tabular}{|c|c|c|c|}
\hline Shortest paths between & $v_{0}$ & $v_{1}$ & $v_{3}$ \\
\hline$v_{2}$ & 1 & 1 & 1 \\
\hline$v_{j}\left(4 \leq j \leq \frac{n}{2}\right)$ & $\left\lceil\frac{j}{2}\right\rceil$ & $\left\lceil\frac{j-1}{2}\right\rceil$ & $\left\lceil\frac{j-3}{2}\right\rceil$ \\
\hline$v_{\frac{n}{2}+1}$ & $\left\lceil\frac{n-2}{4}\right\rceil$ & $\left\lceil\frac{n}{4}\right\rceil$ & $\left\lceil\frac{n-4}{4}\right\rceil$ \\
\hline$v_{\frac{n}{2}+2}$ & $\left\lfloor\frac{n-2}{4}\right\rfloor$ & $\left\lceil\frac{n-2}{4}\right\rceil$ & $\left\lceil\frac{n-2}{4}\right\rceil$ \\
\hline$v_{\frac{n}{2}+3}$ & $\left\lceil\frac{n-6}{4}\right\rceil$ & $\left\lceil\frac{n-4}{4}\right\rceil$ & $\left\lceil\frac{n}{4}\right\rceil$ \\
\hline$v_{j}\left(\frac{n}{2}+4 \leq j \leq n-1\right)$ & $\left\lceil\frac{n-j}{2}\right\rceil$ & $\left\lfloor\frac{n-j+2}{2}\right\rfloor$ & $\left\lfloor\frac{n-j+4}{2}\right\rfloor$ \\
\hline
\end{tabular}

Theorem 3.1. For $n \geq 10$ and $n \equiv 2(\bmod 4), \beta^{\prime}\left(X_{n, 4}\right)=4$. 
Table 6

\begin{tabular}{|c|c|c|c|}
\hline Shortest paths between & $v_{0}$ & $v_{2}$ & $v_{3}$ \\
\hline$v_{1}$ & 1 & 1 & 1 \\
\hline$v_{j}\left(4 \leq j \leq \frac{n}{2}\right)$ & $\left\lceil\frac{j}{2}\right\rceil$ & $\left\lceil\frac{j-2}{2}\right\rceil$ & $\left\lceil\frac{j-3}{2}\right\rceil$ \\
\hline$v_{\frac{n}{2}}+1$ & $\left\lceil\frac{n-2}{4}\right\rceil$ & $\left\lfloor\frac{n}{4}\right\rfloor$ & $\left\lfloor\frac{n-4}{4}\right\rfloor$ \\
\hline$v_{\frac{n}{2}}+2$ & $\left\lfloor\frac{n-2}{4}\right\rfloor$ & $\left\lceil\frac{n}{4}\right\rceil$ & $\left\lceil\frac{n-2}{4}\right\rceil$ \\
\hline$v_{\frac{n}{2}}+3$ & $\left\lceil\frac{n-6}{4}\right\rceil$ & $\left\lceil\frac{n-4}{4}\right\rceil$ & $\left\lceil\frac{n}{4}\right\rceil$ \\
\hline$v_{j}\left(\frac{n}{2}+4 \leq j \leq n-1\right)$ & $\left\lceil\frac{n-j}{2}\right\rceil$ & $\left\lfloor\frac{n-j+2}{2}\right\rfloor$ & $\left\lfloor\frac{n-j+4}{2}\right\rfloor$ \\
\hline
\end{tabular}

Proof. From the definition of fault-tolerant metric dimension it can be seen that $\beta^{\prime}(G) \geq \beta(G)+1[11]$. This implies that $\beta^{\prime}\left(X_{n, 4}\right) \geq 4$ since $\beta\left(X_{n, 4}\right)=3[1]$.

Now for the lower bound, consider the set $W^{\prime}=\left\{v_{1}, v_{2}, v_{3}, v_{4}\right\}$. Since $W$ contains the set $W_{1}$ listed in Theorem 3.1, so $W$ is a resolving set for $X_{n, 4}$. Now we will show that for each $x \in W$, the set $W \backslash\{x\}$ is a resolving set for $X_{n, 4}$. If $x \in\left\{v_{1}, v_{4}\right\}$, then $W \backslash\{x\}$ is a resolving set by setting $i=1$ and $i=2$ in part $(i)$ of Lemma 3.1. If $x=v_{2}$, then by setting $i=1$ in part (iii) of Lemma 3.1, we conclude that $W \backslash\{x\}$ is a resolving set. Finally $\left\{v_{1}, v_{2}, v_{4}\right\}$ is a resolving set of $X_{n, 4}$ by Lemma 3.1 (ii). Therefore $\beta^{\prime}\left(X_{n, 4}\right)=4$

\section{Acknowledgments}

The authors would like to thank the referee for his/her helpful remarks which have contributed to improve the presentation of the article.

\section{R E F E R E N C E S}

1. A. Borchert and S. Gosselin, The metric dimension of circulant graphs and Cayley hypergraphs, To appear in Util. Math.

2. P.S. Buczkowski, G. Chartrand, C. Poisson, P. Zhang, On k-dimensional graphs and their bases, Period. Math. Hungar., 46(1)(2003) 9-15.

3. Z. Beerloiva, F. Eberhard, T. Erlebach. A. Hall, M. Hoffmann, M. Mihalák, L. Ram, Network discovery and verification, IEEE J. Selected Area in Commun., 24(2006) 21682181.

4. G. Chartrand, L. Eroh, M.A. Johnson, O. R. Oellermann, Resolvability in graphs and the metric dimension of a graph, Discrete Appl. Math., 105(2000) 99-113.

5. G. Chartrand, P. Zhang, The theory and applications of resolvability in graphs: A survey, Congr. Numer., 160(2003) 47-68.

6. M.A. Chaudhry, I. Javaid, M. Salman, Fault-Tolerant Metric and Partition Dimension of Graphs, Util. Math., 83(2010) 187-199.

7. C. Godsil and G. Royle, Algebraic graph theory, volume 207 of Graduate Texts in Mathematics, Springer-Verlag, NewYork, (2001).

8. F. Harary, R.A. Melter, On the metric dimension of a graph, Ars. Combin., 2(1976) 191-195. 
9. C. Hernando, M. Mora, P.J. Slater, D.R. Wood, Fault-Tolerant metric dimension of graphs, Proc. Internat. Conf. Convexity in Discrete Structures, Ramanujan Math. Society Lecture Notes, 5(2008) 81-85.

10. I. Javaid, M.T. Rahim and K. Ali, Families of regular graphs with constant metric dimension, Util. Math., 75 (2008) 21-33.

11. I. Javaid, M. Salman, M.A. Chaudhry, S. Shokat, Fault-Tolerance in Resolvability, Util. Math., 80(2009) 263-275.

12. S. Khuller, B. Raghavachari, A. Rosenfeld, Landmarks in graphs, Discrete Appl. Math., 70(1996) 217-229.

13. K. Liu, N. Abu-Ghazaleh, Virtual coordinate back tracking for void travarsal in geographic routing, Lecture Notes Comp. Sci., 4104(2006) 46-59.

14. M. Salman, I. Javaid, M.A. Chaudhry, Resolvability in circulant graphs, Acta Mathematica Sinica, English Series, 9(2012) 1851-1864.

15. P.J. Slater, Leaves of trees, Cong. Numer., 14(1975) 549-559.

Narjes Seyedi

Department of Mathematics

Science and Research Branch, Islamic Azad University

Tehran, Iran

seyedi.narjes@gmail.com

Hamid Reza Maimani

Mathematics Section, Department of Basic Sciences

Shahid Rajaee Teacher Training University

P.O. Box 16785-163

Tehran, Iran

maimani@ipm.ir 